\title{
Unidirectional barbed suture versus standard monofilament for urethrovesical anastomosis during robotic assisted laparoscopic radical prostatectomy
}

\author{
Marc Manganiello, Patrick Kenney, David Canes, Andrea Sorcini, Alireza Moinzadeh \\ Institute of Urology, Lahey Clinic, Burlington, MA, USA
}

\begin{abstract}
Purpose: V-Loc ${ }^{\mathrm{TM}} 180$ (Covidien Healthcare, Mansfield, MA) is a new unidirectional barbed suture that may reduce loss of tension during a running closure. We evaluated the use of the barbed suture for urethrovesical anastomosis (UVA) during robotic assisted laparoscopic prostatectomy (RALP). Time to completion of UVA, post-operative anastomotic leak rate, and urinary incontinence were compared in patients undergoing UVA with 3-0 unidirectional-barbed suture vs. 3-0 Monocryl ${ }^{\mathrm{TM}}$ (Ethicon, Somerville, NJ).

Materials and Methods: Data were prospectively collected for 70 consecutive patients undergoing RALP for prostate cancer between November 2009 and October 2010. In the first 35 patients, the UVA was performed using a modified running van Velthoven anastomosis technique using two separate 3-0 monofilament sutures. In the subsequent 35 patients, the UVA was performed using two running novel unidirectional barbed sutures. At 7-12 days postoperatively, all patients were evaluated with a cystogram to determine anastomotic integrity. Urinary incontinence was assessed at two months and five months by total daily pad usage. Clinical symptoms suggestive of bladder neck contracture were elicited.

Results: Age, PSA, Gleason score, prostate size, estimated blood loss, body mass index, and clinical and pathologic stage between the 2 groups were similar. Comparing the monofilament group and V-Loc ${ }^{\mathrm{TM}} 180$ cohorts, average time to complete the anastomosis was similar (27.4 vs. 26.4 minutes, $p=0.73$ ) as was the rate of urinary extravasation on cystogram ( $5.7 \%$ vs. $8.6 \%, p=0.65$ ). There were no symptomatic bladder neck contractures noted at 5 months of follow-up. At 2 months, the percentage of patients using 2 or more pads per day was lower in the V-Loc ${ }^{\mathrm{TM}} 180$ cohort ( $24 \%$ vs. $44 \%, p<0.02$ ). At 5 months, this difference was no longer evident. Conclusions: Time to complete the UVA was similar in the intervention and control groups. Rates of urine leak were also comparable. While the V-Loc ${ }^{\mathrm{TM}} 180$ was associated with improved early continence, this difference was transient.
\end{abstract}

ARTICLE INFO

\section{Key words:}

sutures; anastomosis;

prostatectomy; robotics

Int Braz J Urol. 2012; 38: 89-96

Submitted for publication:

August 17, 2011

Accepted after revision:

November 29, 2011

\section{INTRODUCTION}

Robotic-assisted laparoscopic prostatectomy (RALP) is a widely utilized, minimally invasive option for the treatment of prostate cancer and, in the short term, has demonstrat- ed similar oncologic outcomes when compared with the open radical prostatectomy $(1,2)$. The urethrovesical anastomosis (UVA) is a technically challenging aspect of RALP (3). Several UVA techniques have been described including a running suture anastomosis, interrupted su- 
ture anastomosis, and use of a Lapra-Ty (Ethicon Endosurgery, Cincinnati, Ohio) to secure the posterior anastomosis $(4,5)$. Drawbacks to these techniques include slippage and relaxation of the suture and Lapra-Ty clip erosion. Recently, an absorbable unidirectional barbed suture has become available (V-Loc ${ }^{\mathrm{TM}} 180$, Covidien, Mansfield, MA) (Figure-1). This suture has been used for skin and soft tissue closures, gynecologic procedures, and bowel anastomosis in a porcine rate, symptomatic bladder neck contractures, and urinary continence in patients undergoing RALP with barbed versus non-barbed suture.

\section{MATERIALS AND METHODS}

We assessed 70 consecutive patients undergoing RALP for prostate cancer by one surgeon over a 10-month period. Prior to enrollment and prospective data collection, the Institutional

Figure 1 - V-Loc ${ }^{\text {TM }} 180$ suture.

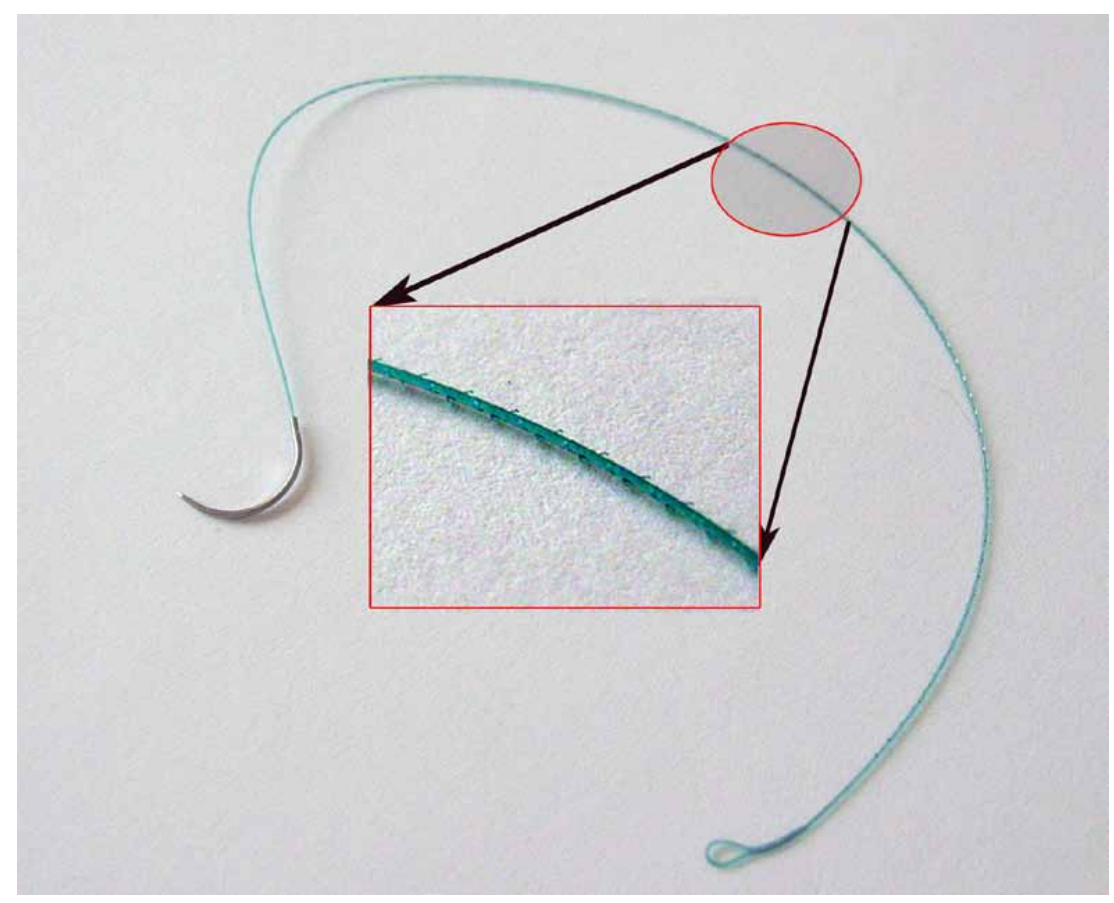

model (6-8). Anastomoses using standard monofilament for running closure requires frequent re-tightening, with some surgeons relying on the assistant to follow the suture to maintain tension. Anchoring barbs have the potential to obviate many of these maneuvers, as the barbs may minimize loosening of the suture and allow for optimal cooptation of the urethra and bladder neck. In fact, the need for an assistant during the UVA may be obviated with the barbed suture. We sought to evaluate UVA time, urine leak
Review Board approved data collection for our radical prostatectomy patients. Patients were recruited during routine evaluation for their prostate cancer. Inclusion criteria included clinically organ confined or locally advanced prostate cancer (clinical $\leq \mathrm{T} 3 \mathrm{a}$ ). Exclusion criteria included a history of chronic steroid use, severe vascular disease, or evidence of gross disease invading the bladder neck at the time of surgery.

In the first 35 patients, the UVA was performed using a modified Van Velthoven tecnique 
(5). Briefly, two separate 3-0 monocryl sutures cut to 7 inches with a Lapra-Ty on the distal end were used to perform a running anastomosis. The first suture was run clockwise from 6 o'clock to 12 o'clock. The second was run counter clockwise from 5 o' clock to 12 o'clock. The two sutures were tied to each other at 12 o'clock with the knot resting on the exterior of the anastomosis.

In the subsequent 35 patients, the UVA was performed with the 3-0 unidirectional absorbable barbed suture. This anastomotic suture is designed such that the 3 centimeters distal to the needle are non-barbed. This allows the surgeon to remove the suture in the event the initial throw is suboptimal. The remainder of the suture is barbed. The tail-end of the suture is a loop that is used to anchor the stitch at the bladder neck without a knot. The stitch is anchored at the 6 o'clock position on the bladder neck. The needle is then passed through the 6 o'clock position on the bladder neck followed by the urethra. This is continued clockwise for three to four passes. The suture is then tightened to approximate the urethrovesical anastomosis. Care is taken not to pull these sutures too tight, allowing optimal cooptation of the urethra and bladder neck. This suture is then run in a clockwise fashion towards the 12 o'clock position. A second unidirectional barbed suture is anchored at the 5 o'clock position and run counterclockwise. The second suture is cinched prior to tying the two sutures together. In all cases, the anastomosis is tested for leak intraoperatively with retrograde filling of the bladder with $120 \mathrm{cc}$ of sterile irrigant. A $10 \mathrm{~mm}$ flat Jackson Pratt drain (JP) was inserted in all patients. All patients with JP fluid output greater than $75 \mathrm{cc}$ over an 8 hour shift had fluid sent for creatinine.

Anastomotic time was recorded; starting once the anchoring suture was placed and finished when the two running sutures were tied together. At 7 to 12 days postoperatively (depending on surgical date and date of surgeon's clinic schedule), all patients underwent evaluation with a cystogram to evaluate for anastomotic leak. Those patients without leak had their catheters removed. If a significant anastomotic leak was detected, catheter drainage was continued with a follow-up study scheduled within 2 weeks. Postoperative urinary incontinence was assessed at both the 2 and 5-month follow-up visits by patient reported total daily pad usage during post operative follow-up visits. Endpoints between groups were compared using student t test, log rank test, and fisher's exact test as appropriate.

\section{RESULTS}

Patient age, body mass index, PSA, Gleason score, clinical and pathologic stage, prostate size, positive margin rates, estimated blood loss, and transfusion rates were comparable between the barbed and non-barbed cohorts (Table-1). No patient was excluded based on the exclusion criteria as outlined above. Time to completion of the UVA was not significantly different between the monofilament suture group (avg. 26.6, range 11-51 minutes) when compared to the barbed suture group (avg. 24.8, range 11-55 minutes), $\mathrm{p}=$ 0.51 . All drain creatinine values were consistent with serum and all JPs were removed before hospital discharge.

There was no detectable difference in the rate of radiographic urine leak between the barbed and non-barbed groups (8.6\% vs. 5.7\%, $p=0.65)$. There were no significant differences between groups in terms of total catherization time (Table-1).

None of the patients in either group experienced symptomatic bladder neck contracture. At 2 months, overall pad usage was significantly lower in the V-Loc ${ }^{\mathrm{TM}} 180$ cohort $(\mathrm{p}=0.035)$ and the percentage of patients using 2 or more pads per day was lower in the V-Loc ${ }^{\mathrm{TM}} 180$ cohort $(24 \%$ vs. $44 \%$ ). At 2 months, $76 \%$ of V-Loc ${ }^{\mathrm{TM}} 180$ patients reported using 0 or 1 pad per day, as compared to $56 \%$ of non-barbed patients.

Overall pad usage at 5 months was similar between groups. In both cohorts, $88 \%$ of patients reported using 0 to 1 pads per day, with $12 \%$ reporting 2 or more pads per day. One patient who was incontinent at baseline was excluded from the 2 and 5 month continence analyses, as were two patients for whom complete continence data was not available. 
Table 1 - Comparison of Patients undergoing UVA with Monofilament vs. Barbed Suture.

\begin{tabular}{|c|c|c|c|}
\hline & $\begin{array}{c}\text { Monocryl suture, } \\
\mathrm{n}=35\end{array}$ & $\begin{array}{l}\text { Barbed suture } \\
\quad n=35\end{array}$ & $P$ Value \\
\hline Age & $61.2(48.2-71.5)$ & $58.7(46.6-73.4)$ & 0.1 \\
\hline Body Mass Index (kg/m²) & $28.7(19.3-40.5)$ & $29.0(19.7-40.5)$ & 0.75 \\
\hline PSA & $9.3(0.7-69)$ & $6.4(0.8-13.3)$ & 0.2 \\
\hline Preoperative Gleason Score Sum & $7(6-9)$ & $7(6-9)$ & 0.78 \\
\hline Mean Pathologic prostate size (gm) & $44.2(27-68)$ & $43.5(20-110)$ & 0.84 \\
\hline Mean Estimated Blood Loss (cc) & $125(25-450)$ & $159(50-300)$ & 0.42 \\
\hline Blood Transfusion Rate (cc) & 0 & 0 & $\mathrm{~N} / \mathrm{A}$ \\
\hline Mean Catheterization time (days) & $12.5(8-28)$ & $12.2(8-39)$ & 0.80 \\
\hline \multicolumn{4}{|l|}{ Clinical Stage } \\
\hline T1c & 21 of $35(60 \%)$ & 23 of $35(66 \%)$ & 0.63 \\
\hline $\mathrm{T} 2$ & 14 of $35(40 \%)$ & 12 of $35(34 \%)$ & 0.63 \\
\hline \multicolumn{4}{|l|}{ Pathologic Stage } \\
\hline $\mathrm{T} 2$ & 28 of $35(80 \%)$ & 29 of $35(83 \%)$ & 0.76 \\
\hline T3 & 7 of $35(20 \%)$ & 6 of $35(17 \%)$ & 0.76 \\
\hline Overall Margin + Rate & 5 of $35(14 \%)$ & 2 of $35(6 \%)$ & 0.24 \\
\hline T2 & 1 of $28(3.6 \%)$ & 1 of $29(3.4 \%)$ & 0.98 \\
\hline T3 & 4 of $7(57.1 \%)$ & 1 of $6(16.7 \%)$ & 0.15 \\
\hline Cystogram leak rate & $2(5.7 \%)$ & $3(8.6 \%)$ & 0.65 \\
\hline $\begin{array}{l}\text { Mean Time to completion of the anastomosis } \\
\text { (minutes) }\end{array}$ & $26.6(11-51)$ & $24.8(11-55)$ & 0.51 \\
\hline \multicolumn{4}{|l|}{2 month continence rate } \\
\hline \multicolumn{4}{|l|}{ \# pads per day: } \\
\hline $0-1$ pads/day & 19 of $34(56 \%)$ & 25 of $33(76 \%)$ & 0.035 \\
\hline$>2$ pads/day & 15 of $34(44 \%)$ & 8 of $33(24 \%)$ & \\
\hline \multicolumn{4}{|l|}{5 month continence rate } \\
\hline \multicolumn{4}{|l|}{ \# pads per day: } \\
\hline $0-1$ pads/day & $30 / 34(88 \%)$ & 29/33 (88\%) & 0.411 \\
\hline$>2$ pads/day & $4 / 34(12 \%)$ & $4 / 33(12 \%)$ & \\
\hline
\end{tabular}




\section{DISCUSSION}

The urethrovesical anastomosis is a critical step in RALP and may play a role in urine leak, bladder neck contracture, continence, and duration of catheterization. Unidirectional barbed suture may maintain tension during a running closure such as the UVA, and may therefore reduce urine leak and bladder neck contracture. In our cohort of patients, all anastomoses were checked intraoperatively for watertight closure. There were no instances of urinary leak on intraoperative testing of the urethrovesical anastomosis, suggesting good tissue approximation. There was also no detectable difference in cystographic urine leak between the barbed and non-barbed cohorts (8.6\% vs. 5.7\%, $\mathrm{p}=0.65)$.

In general, published rates of urine leak in RALP range from 0 to $12.7 \%$ (9-12). One series of 2500 patients undergoing RALP by a single surgeon reported the anastomotic leak rate of $1.40 \%$ (3). Our cystographic leak rate was 8.6\% and $5.7 \%$ in the monocryl versus barbed suture group respectively. In another series of UVA performed with barbed suture, Williams et al report a significantly higher incidence of urine leak in the barbed group ( $20 \%$ vs. $2.8 \%, p=0.019)$ and, consequently, a longer mean catheterization time (11.1 days vs. 8.3 days, $p=0.048$ ). Our data is consistent with other published studies and argues against an association between barbed suture and anastomotic leak, although it is possible that our sample size was too small to detect a very small difference in urine leak. However, this small difference would most likely be clinically insignificant given our relative low percentage of leak. Studies have shown that a majority (79 to 95\%) of patients undergoing radical prostatectomy do not have a urine leak by cystogram seven days postoperatively (13-15). By 15 to 21 days after surgery, nearly $100 \%$ of patients do not have a urinary leak at the UVA $(13,16)$. The type or number of sutures used has not seemed to affect urinary leak rates, suggesting that the most important factor in a leak-free UVA is good tissue approximation and an intraoperative watertight closure.

In the contemporary literature, bladder neck contractures are reported in 0 to $7.4 \%$ of
RALP patients and in 2.6 to $9 \%$ of patients after open radical prostatectomy $(9,10,12,17,18)$. The exact cause of bladder neck contracture is not known although several theories exist. These potential hypotheses include increased operative blood loss, poor mucosal approximation during UVA, ischemia of bladder neck tissue, extended length of urinary catheterization, urinary infection, and urinary extravasation. However, no study has demonstrated with significance any of these variables leading to a bladder neck contracture (18-20). Webb et al. found a significant difference in the number of bladder neck contractures between patients undergoing RALP vs. open radical prostatectomy ( 0 vs. $9 \%, p<0.005$ ). This was attributed to difference in technique since the open procedure requires bladder neck stomatization and mucosal eversion (18). With excessive tension on the urethrovesical anastomosis, future tissue ischemia may develop with subsequent fibrosis and scarring, leading to bladder neck contracture. The unidirectional sutures may aide in improved tissue handling and decrease the tension placed on the running suture during the anastomosis while re-approximating the urethra and bladder neck. Msezane et al. reported that patients who ultimately developed a bladder neck contracture had significantly longer operative times ( 283 vs. $225 \mathrm{~min}, \mathrm{p}=0.04$ ). All other patient and operative variables, however, were equal (20). In the present study, there were no symptomatic bladder neck contractures detected in either group. This may again be a function of the relatively small sample size and short followup. In addition, it is possible that there were asymptomatic bladder neck contractures that went undetected. In order to assess for bladder neck contracture, all patients would have needed to be submitted to cystoscopy and uroflow studies which were not completed in this study.

Although it is conceivable that unidirectional suture may reduce the time to completion of the UVA given reduced time spent keeping the suture from slipping, no difference was observed. In other published series, time to completion of the anastomosis was faster using barbed versus nonbarbed suture (8 to 19 minutes faster) (21-23). In our series, the lack of difference may be due to 
resident involvement in operative cases, as our institution is a teaching hospital. Under the guidance of the attending surgeon, the chief resident may perform a portion of the procedure. As such, the anastomosis time is not representative of one single surgeon, and will vary.

Several groups have recently compared the unidirectional barbed suture to the standard monocryl suture in the UVA during RALP. Only immediate perioperative data have been assessed in these studies and as such 2 and 5 month follow-up for continence or bladder neck contractures are not reported (21-24). In the comparative studies, no data is provided for urinary incontinence between patients undergoing the UVA with barbed versus standard monofilament suture. However, theories on the potential improvements of barbed suture versus monofilament suture were discussed. Moran et al. stated that a bidirectional barbed suture allows for minimal tension during the UVA, resulting in less inflammation, scar formation, and tissue ischemia (24).

Urinary incontinence is one of the most common long term complications following RALP. A large series of $>2700$ men undergoing RALP reported a minimum of 12 months (mean of 28 months, complete data on 1110 patients) follow-up as regards their post-operative urinary function (25). Overall, 93\% (1032 of 1110) of patients were wearing less than or equal to 1 pad per day at last follow-up for a mean of 28 months (range 12 to 71 months); $23.7 \%$ actually felt they had complete continence at the time of catheter removal (mean post-operative day 7 to 8 ). Our 5 month reported $\leq 1$ pad usage of $88 \%$ in each group compares favorably with other published series. However, patient reports of daily pad usage are subjective and dependent on individual preference as to when a pad should be changed. No difference was noted in our series. We did not objectively calculate the amount of urine leakage a patient experienced. This may have been performed by recording differences in pad weight rather than differences in number of daily pads used. The measurement of pad weight for quantifying urine leakage is certainly a superior method to pad number, and we acknowledge this as a lim- itation of our study. However, it is likely that continence is determined to a greater degree by patient's baseline function, precise apical dissection that respects the pelvic floor anatomy, the degree of bladder neck preservation, nerve sparing and other as yet undetermined factors other than the technical nuances of the anastomosis itself (26).

While data driven aspects of this suture are important, the surgeon's subjective impression also needs consideration, as other authors have recognized. Kaul et al. reported the UVA using barbed suture was easier than standard monofilament, an important consideration especially for the inexperienced robotic surgeon. The barbed suture prevents slippage. Often an assistant provides intermittent traction between each throw with the non barbed running anastomosis to prevent inadvertent slippage. The need for an assistant in this regard is eliminated, minimizing instrument clashes and potentially excessive tension placed on the anastomosis by the assistant. Lapra-Ty has been used in the past to help secure the posterior urethral reconstruction; however, with the use of barbed suture, the erosion of dissolvable clips is eliminated.

We currently prefer the V-Loc ${ }^{\mathrm{TM}} 180$ suture for RALP anastomosis and use it exclusively despite the lack of objective endpoints evaluated in this manuscript. This suture obviates the need for an assistant to follow the suture to continually reapply tension to previous throws. Once the bladder neck and urethral tissue are re-approximated, the tissue stays in place and does not migrate unless there is significant counter tension (which is rare). Although not reported in patients in this series, based on our prior experience, use of Lapra-Ty as the anchor for monocryl sutures leads to $\sim 1 \%$ rate of clip erosion with subsequent expulsion during voiding. The primary surgeon in this study prefers the modification of the Van Velthoven technique of using 2 separate sutures anchored to the bladder neck rather than tied together. As such, the two sutures do not interfere with one another during the anastomosis. Alternatively, the two unidirectional sutures may be passed through the loop of one another to perform a classic Van Velthoven technique. 


\section{CONCLUSIONS}

Our findings demonstrate that time to completion of the UVA as well as postoperative urine leak rates on cystogram were similar between a barbed or standard monofilament suture. Subjective urinary incontinence at 2 months for patients with the barbed suture was improved when compared to those who underwent the anastomosis with monocryl. The clinical significance of this in a small sample size is unclear. At 5 months, this significance was no longer demonstrated.

\section{CONFLICT OF INTEREST}

None declared.

\section{REFERENCES}

1. Hakimi AA, Feder M, Ghavamian R: Minimally invasive approaches to prostate cancer: a review of the current literature. Urol J. 2007; 4: 130-7.

2. Herrmann TR, Rabenalt R, Stolzenburg JU, Liatsikos EN, Imkamp F, Tezval $\mathrm{H}$, et al.: Oncological and functional results of open, robot-assisted and laparoscopic radical prostatectomy: does surgical approach and surgical experience matter? World J Urol. 2007; 25: 149-60.

3. Coelho RF, Palmer KJ, Rocco B, Moniz RR, Chauhan S, Orvieto MA, et al:: Early complication rates in a singlesurgeon series of 2500 robotic-assisted radical prostatectomies: report applying a standardized grading system. Eur Urol. 2010; 57: 945-52.

4. Ball AJ, Bordeau KP, Davis JW, Given RW, Lynch DF, Fabrizio MD: Modified running vesicourethral anastomosis after robotically assisted laparoscopic radical prostatectomy: use of solitary Lapra-Ty to secure posterior approximation. Urology. 2005; 66: 16-8.

5. Van Velthoven RF, Ahlering TE, Peltier A, Skarecky DW, Clayman RV: Technique for laparoscopic running urethrovesical anastomosis:the single knot method. Urology. 2003; 61: 699-702.

6. Demyttenaere SV, Nau P, Henn M, Beck C, Zaruby J, Primavera M, Kirsch D, Miller J, Liu JJ, Bellizzi A, Melvin WS. Barbed suture for gastrointestinal closure: a randomized control trial. Surg Innov. 2009; 16: 237-42.

7. Einarsson JI, Chavan NR, Suzuki Y, Jonsdottir G, Vellinga TT, Greenberg JA: Use of bidirectional barbed suture in laparoscopic myomectomy: evaluation of perioperative outcomes, safety, and efficacy. J Minim Invasive Gynecol. 2011; 18: 92-5.
8. Shermak MA, Mallalieu J, Chang D: Barbed suture impact on wound closure in body contouring surgery. Plast Reconstr Surg. 2010; 126: 1735-41.

9. Rebuck DA, Haywood S, McDermott K, Perry KT, Nadler $\mathrm{RB}$ : What is the long-term relevance of clinically detected postoperative anastomotic urine leakage after roboticassisted laparoscopic prostatectomy? BJU Int. 2011; 108: 733-8.

10. Sammon JD, Muhletaler F, Peabody JO, Diaz-Insua M, Satyanaryana R, Menon M: Long-term functional urinary outcomes comparing single- vs double-layer urethrovesical anastomosis: two-year follow-up of a two-group parallel randomized controlled trial. Urology. 2010; 76: 1102-7.

11. Shah G, Vogel F, Moinzadeh A: Nephroureteral stent on suction for urethrovesical anastomotic leak after robot-assisted laparoscopic radical prostatectomy. Urology. 2009; 73: $1375-6$.

12. Shalhav AL, Orvieto MA, Chien GW, Mikhail AA, Zagaja GP, Zorn KC: Minimizing knot tying during reconstructive laparoscopic urology. Urology. 2006; 68: 508-13.

13. Eggert T, Palisaar J, Metz P, Noldus J: Assessing the vesico-urethral anastomosis after radical retropubic prostatectomy: transrectal ultrasonography can replace cystography. BJU Int. 2007; 100: 1268-71.

14. Guru KA, Seereiter PJ, Sfakianos JP, Hutson AD, Mohler $\mathrm{JL}$ : Is a cystogram necessary after robot-assisted radical prostatectomy? Urol Oncol. 2007; 25: 465-7.

15. Varkarakis J, Wirtenberger W, Pinggera GM, Berger A, Harabayashi T, Bartsch G, et al.: Evaluation of urinary extravasation and results after continence-preserving radical retropubic prostatectomy. BJU Int. 2004; 94: 991-5.

16. Poulakis V, Skriapas K, de Vries R, Dillenburg W, Witzsch $U$, Becht E: Vesicourethral anastomosis during endoscopic extraperitoneal radical prostatectomy: a prospective comparison between the single-knot running and interrupted technique. Urology. 2006; 68: 1284-9.

17. Breyer BN, Davis CB, Cowan JE, Kane CJ, Carroll PR: Incidence of bladder neck contracture after robot-assisted laparoscopic and open radical prostatectomy. BJU Int. 2010; 106: 1734-8.

18. Webb DR, Sethi K, Gee K: An analysis of the causes of bladder neck contracture after open and robot-assisted laparoscopic radical prostatectomy. BJU Int. 2009; 103: 957-63.

19. Hanson GR, Odom E, Borden LS Jr, Neil N, Corman JM: Post-operative drain output as a predictor of bladder neck contracture following radical prostatectomy. Int Urol Nephrol. 2008; 40: 351-4.

20. Msezane LP, Reynolds WS, Gofrit ON, Shalhav AL, Zagaja GP, Zorn KC: Bladder neck contracture after robot-assisted laparoscopic radical prostatectomy: evaluation of incidence and risk factors and impact on urinary function. J Endourol. 2008; 22: 97-104. 
21. Kaul S, Sammon J, Bhandari A, Peabody J, Rogers CG, Menon M: A novel method of urethrovesical anastomosis during robot-assisted radical prostatectomy using a unidirectional barbed wound closure device: feasibility study and early outcomes in 51 patients. J Endourol. 2010; 24: 1789-93.

22. Tewari AK, Srivastava A, Sooriakumaran P, Slevin A, Grover $S$, Waldman 0 , et al.: Use of a novel absorbable barbed plastic surgical suture enables a "self-cinching" technique of vesicourethral anastomosis during robot-assisted prostatectomy and improves anastomotic times. J Endourol. 2010; 24: 1645-50.

23. Williams SB, Alemozaffar M, Lei Y, Hevelone N, Lipsitz SR, Plaster BA, et al.: Randomized controlled trial of barbed polyglyconate versus polyglactin suture for robot-assisted laparoscopic prostatectomy anastomosis: technique and outcomes. Eur Urol. 2010; 58: 875-81.

24. Moran ME, Marsh C, Perrotti M: Bidirectional-barbed sutured knotless running anastomosis v classic Van Velthoven suturing in a model system. J Endourol. 2007; 21: 1175-8.
25. Badani KK, Kaul S, Menon M: Evolution of robotic radical prostatectomy: assessment after 2766 procedures. Cancer. 2007; 110: 1951-8.

26. Moinzadeh A, Shunaigat AN, Libertino JA: Urinary incontinence after radical retropubic prostatectomy: the outcome of a surgical technique. BJU Int. 2003; 92: 355-9.
Correspondence address:

Dr. Alireza Moinzadeh Institute of Urology

Lahey Clinic

41 Mall Road

Burlington, MA, 01805, USA

Fax: + 1781 744-5429

E-mail: moinza00@lahey.org 